\title{
The endophytic fungi of Salvia miltiorrhiza Bge.f. alba are a potential source of natural antioxidants
}

\author{
Yan-Ling $\mathrm{Li}^{1 \dagger}$, Xiao-Ming Xin ${ }^{2 \dagger}$, Zheng-Yao Chang ${ }^{1}$, Ren-Jiu Shi ${ }^{1 *}$, Zeng-Min Miao ${ }^{1}$, Jing Ding ${ }^{3}$ \\ and Gang-Ping Hao ${ }^{1 *}$
}

\begin{abstract}
Background: Salvia miltiorrhiza Bge. f. alba is a traditional Chinese herbal drug with special pharmacological effect on thromboangiitis obliterans. However, the nature source of S.miltiorrhiza Bge.f.alba is now in short supply because of the over-collection of the wild plant. To better utilize this resource, the diversity and antioxidant activity of endophytic fungi isolated from S. miltiorrhiza Bge. f. alba were investigated.

Results: A total of 14 endophytic fungi were isolated from different parts of S. miltiorrhiza Bge.f.alba. Based on morphological and molecular identification, the endophytic fungi isolated were classified into four genera (Alternaria sp., Fusarium sp., Schizophyllum sp. and Trametes sp.). These fungal extracts were prepared using ethanol and evaluated for their phytochemical compounds and antioxidant activity. Alternaria alternata SaF-2 and Fusarium proliferatum SaR-2 are of particular interest because they yielded all of nine phytochemicals including saponins, phenol, flavonoids, cardiac glycosides, steroids, tannins, alkaloids, anthroquinone and terpenoids. F. proliferatum SaR-2 and A. alternata SaF-2 also exhibited stronger antioxidant activities by FRAP and DPPH method, having the higher levels of phenol and flavonoid than those of plant root. The total amount of phenol and flavonoid quantified were of 21.75, 20.53 gallic acid equivalent per gram and 8.27 and $7.36 \mu \mathrm{g} / \mathrm{mg}$ of quercetin equivalent respectively. These two endophytic fungi (SaR-2 and SaF-2) were found to have comparable scavenging abilities on both FRAP (1682.21 and $1659.05 \mu \mathrm{mol} / \mathrm{mg}$, respectively) and DPPH-free radicals ( $90.14 \%$ and $83.25 \%$, respectively, at $0.1 \mathrm{mg} / \mathrm{mL}$ ). This is the first report about isolation of endophytic fungi from S. miltiorrhiza Bge.f.alba and their antioxidant activities.
\end{abstract}

Conclusions: These results indicate that the endophytic fungi associated with S. miltiorrhiza Bge.f. alba can be a potential source of novel natural antioxidants.

Keywords: Salvia miltiorrhiza Bge.f.alba; Endophytic fungi; Identification; Phytochemicals; Antioxidant activity

\section{Background}

Salvia miltiorrhiza Bunge is a well-known medicinal plant, and its root, called "dan shen" in Chinese, is a traditional Chinese herbal drug used for the treatment of various kinds of diseases, especially for cardiovascular and cerebrovascular diseases (Zhou et al. 2012). S. miltiorrhiza Bge.falba is a white flowered varietas of S. miltiorrhiza Bunge and was present only in Shandong province of China. Studies showed that it had special pharmacological effect on thromboangiitis obliterans (Hao et al. 2009). The main bioactive constituents in root of two kinds of S. miltiorrhiza include lipid-soluble diterpenes (dihydrotanshione I, tanshinone I,

\footnotetext{
* Correspondence: rjshi@tsmc.edu.cn; haogangping@163.com

${ }^{\dagger}$ Equal contributors

${ }^{1}$ College of Life Sciences, Taishan Medical University, Tai'an 271016, China

Full list of author information is available at the end of the article
}

cryptotanshinone and tanshinone IIA), and water-soluble phenolic compounds (salvianolic, rosmarinic, salvianic acid and protocatechuic) (Chen and Chen 1999; Liu et al. 2007). Previous research on phenolic compounds showed that there were important biological activities such as antioxidant and antithrombotic effects (Lam et al. 2007; Li 1997; Yan et al. 2006; Zhou et al. 2005). Compared with S. miltiorrhiza Bunge, S. miltiorrhiza Bge.falba has higher phenolic acids contents and higher pharmaceutical values with potential application in pharmaceutical industry (Hao et al. 2009; Hao et al. 2012). However, the nature source of S.miltiorrhiza Bge.f.alba is now in short supply because of the over-collection of the wild plant. Therefore, it is important to find a substitutable approach to produce the active compounds similar with the host plant to meet the medical demand. 
Plant endophytic fungi are symbiotic fungi that inhabit the interior of the healthy tissues of the host plants without causing apparent symptoms of disease (Saikkonen et al. 1998). They have been found in every plant species examined, and it is estimated to be around over one million endophytic fungi colonizing in plants. Endophytic fungi residing within these plants are able to produce bioactive compounds such as paclitaxel, podophyllotoxin and camptothecine (Aly et al. 2010), which were also produced by their respective host plants. This is advantageous for us to develop an alternative way for efficiently producing these valuable and scarce bioactive constituents. Thus plant endophytic fungi have been considered to be a novel and promising resource of natural bioactive compouds with extensive application in agriculture, industry and medicine field (Schulz et al. 2002; Strobel et al. 2004; Verma et al. 2009). Many valuable bioactive products from endophytes have been recognized as promising sources of antimicrobial, antioxidant, and anticancer substances (Jayanthi et al., 2011; Lin et al. 2007; Sadananda et al. 2011). These bioactive products could be classified as saponins, phenol, flavonoids, cardiac glycosides, steroids, tannins, alkaloids, anthroquinone and terpenoids (Tan and Zou 2001; Zhang et al. 2006).

Increasing evidence showed that reactive oxygen species (ROS) could cause oxidative damage of lipids, proteins, DNA and RNA, eventually enhancing the risk for aging, cardiovascular disease, cancer, atherosclerosis, diabetes, Alzheimer's disease and other diseases (Finkel and Holbrook 2000; Lachance et al. 2001). Therefore, antioxidants are believed to be highly effective radical scavengers in the prevention of this ROS mediated diseases. To date, there is little report about isolation and antioxidant activities of endophytic fungi from S. miltiorrhiza Bge.falba. The present study, therefore, was carried out to better understand the phytochemicals and antioxidant potential of endophytic fungi from S. miltiorrhiza Bge.falba.

\section{Methods}

\section{Plant materials}

Nine healthy Salvia miltiorrhiza Bge. f. alba were randomly collected in July 2010 from Salvia miltiorrhiza cultivation zone at three sites in Taishan Medical University, Shandong Province, China. The current-year roots (4-6 cm $\times 2-3 \mathrm{~cm}$, length $\times$ diameter), stems $(8-12 \mathrm{~cm} \times$ $1-2 \mathrm{~cm}$, length $\times$ diameter), leaves and flowers were placed in sterile plastic bags and stored at $4^{\circ} \mathrm{C}$ until isolation procedure was started.

\section{Isolation of fungal endophytes}

Plant tissues were surface-sterilized using the procedure described by Fisher et al. (1993) with minor modification. The root, stem, leaf and flower segments were cleaned under running tap water. After air-drying, the cleaned stems and roots were cut into small pieces, and then all the tissues were surface sterilized by immersion in $75 \%$ ethanol for $1 \mathrm{~min}$, 5\% sodium hypochlorite solution for $3 \mathrm{~min}$ and then washed three times in sterile distilled water for $1 \mathrm{~min}$ each time. The surface-sterilized samples were cut into small pieces using a sterile blade and placed on sterile potato dextrose agar (PDA) at $28^{\circ} \mathrm{C}$. The hyphal tip of endophytic fungus growing out from the plant tissue was cut by a sterile pasture pipette and transferred to PDA plate supplemented with ampicillin $(150 \mathrm{mg} / \mathrm{L})$ and streptomycin $(100 \mathrm{mg} / \mathrm{L})$ to inhibit the bacterial growth until the mycelium or colony appeared surrounding the segments. The efficiency of surface sterilization procedure was checked for each sterilized plant segment following the imprint method. In addition, to check the presence of surface associated fungi, plant samples without surface sterilization were cultured in the same condition as negative controls. Colonization frequency (CF) was calculated as the total number of plant tissue segments colonized by an endophyte, divided by the total number of incubated segments.

Endophytic fungi from the roots, stems, leaves and flowers of Salvia miltiorrhiza Bge. f. alba have been numbered and codified as SaR1-SaR7, SaS1, SaL1 and SaF1- SaF5. All the isolated endophytic fungi have been stored on PDA slants at $4^{\circ} \mathrm{C}$ and kept at College of Life Sciences, Taishan Medical University, Shandong Province, China.

\section{Identification of fungal endophytes}

The identification procedure of endophytic fungi was based on morphology and molecular methods. The morphological characters included culture characteristics and the morphology of conidia. The molecular method was carried out to characterize some non-sporulating group using the ribosomal internal transcribed spacer (ITS) sequence. Fungal genomic DNA was extracted from fresh mycelia using an SDS extraction protocol described by Plaza et al. (2004). The primers ITS1 (5'-TCCGTAGGT GAACCTGCGG-3') and ITS4 (5'-TCCTCCGCTTATT GATATGC-3') were used to amplify the ITS region. The PCR products were subsequently purified and sequenced in two directions on an ABI 3700 automated sequencer. The resulting sequences were subjected to BLAST searches of the NCBI GenBank database to determine the identity of the fungi.

\section{Fermentation and preparation of crude extracts}

Each isolated endophytic fungus strain was inoculated in potato dextrose liquid medium with $150 \mathrm{rpm}$ shaking at $25^{\circ} \mathrm{C}$ for $5-7$ days. Mycelia and broths were separated by filteration through two layers of cheesecloth. Mycelia were thoroughly washed with sterile distilled water, airdried in an oven at $60^{\circ} \mathrm{C}$, and ground into fine powder. 
The dried mycelia sample (2 g) was extracted with $50 \mathrm{~mL}$ of $95 \%$ ethanol for three times. Culture filtrates was treated by rotary evaporation under vacuum and then extracted thrice with a threefold of the volume of $95 \%(\mathrm{v} / \mathrm{v})$ ethanol. The obtained extracts were filtered by Whatman No.1 filter paper and then concentrated under vacuum at $45^{\circ} \mathrm{C}$ to yield the crude extracts. As a control, $2 \mathrm{~g}$ of plant root power was extracted with $50 \mathrm{~mL}$ of $95 \%$ ethanol and stored at $4^{\circ} \mathrm{C}$ till further process.

\section{Phytochemical screening}

Preliminary phytochemical analysis of the crude extracts of fungi and plant root was carried out for the presence of the following metabolites such as saponins, phenol, flavonoids, cardiac glycosides, steroids, tannins, alkaloids, anthroquinone and terpenoids according to standard methods (Devi et al. 2012; Edeoga et al. 2005; Maobe et al. 2013).

\section{Determination of total phenolic content}

Total phenolic contents from endophytic fungi and plant root were respectively measured by Folin-Ciocalteu's colorimetric method (Taga et al. 1984) with some modification. Briefly, $100 \mathrm{mg}$ of different ethanol extracts was added to $5 \mathrm{~mL}$ of $0.3 \% \mathrm{HCl}$ in methanol/deionised water $(60: 40, \mathrm{v} / \mathrm{v})$ respectively. The resulting mixture $(100 \mu \mathrm{L})$ was added to $2 \mathrm{~mL}$ of $2 \%$ aqueous sodium carbonate. Then the mixture was incubated for $2 \mathrm{~min}$ at room temperature. $100 \mu \mathrm{L}$ of $50 \%$ Folin-Ciocalteu's reagent was added to treated mixture and incubated for $30 \mathrm{~min}$ at room temperature, and absorbance was measured at $750 \mathrm{~nm}$ with the spectrophotometer against blank. The total phenol content was calculated on the basis of the standard curve of gallic acid. Phenol contents were expressed as $\mathrm{mg}$ of gallic acid equivalents (GAEs) per g of extract.

\section{Determination of total flavonoid content}

Total flavonoid content was estimated by a colorimetric method reported by Barros et al. (2007). The extract $(250 \mu \mathrm{L})$ was mixed with distilled water $(1.25 \mathrm{~mL})$ and sodium nitrite solution $(5 \%, 75 \mu \mathrm{L})$. After 5 min incubation at room temperature, aluminum chloride solution $(10 \%, 150 \mu \mathrm{L})$ was added. After $6 \mathrm{~min}$, sodium hydroxide $(1 \mathrm{M}, 500 \mu \mathrm{L})$ and distilled water $(275 \mu \mathrm{L})$ were added to the mixture. The solution was mixed well and incubated at $25^{\circ} \mathrm{C}$ for $30 \mathrm{~min}$. Absorbance was measured at $510 \mathrm{~nm}$ against blank. The content of flavonoid was calculated on the basis of the standard curve of quercetin and the results were expressed as mg of quercetin equivalents per $g$ of extract.

\section{Determination of antioxidant activity}

Antioxidant activity of examined extracts was measured using ferric ion reducing antioxidant power (FRAP) and 1,1-diphenyl-2-picryl-hydrazyl (DPPH) assay.

\section{FRAP assay}

FRAP reagents was freshly prepared by mixing $25 \mathrm{~mL}$ acetate buffer (300 mM, pH 3.6), $2.5 \mathrm{~mL} \mathrm{2,4,6-tris} \mathrm{(2-}$ pyridyl)-S-triazine (TPTZ) solution (10 $\mathrm{mM}$ TPTZ in $40 \mathrm{mM} / \mathrm{L} \mathrm{HCl})$ and $2.5 \mathrm{~mL} \mathrm{FeCl}_{3}(20 \mathrm{mM})$ water solution. Each sample $(150 \mu \mathrm{L})(0.5 \mathrm{mg} / \mathrm{mL})$ dissolved in methanol was added to $4.5 \mathrm{~mL}$ of freshly prepared FRAP reagent and stirred. After $5 \mathrm{~min}$, absorbance was measured at $593 \mathrm{~nm}$, using FRAP working solution as blank (Szőllősi and Szőllősi Varga 2002; Tomic et al. 2009). A calibration curve of ferrous sulfate $(100-1000 \mu \mathrm{mol} / \mathrm{L})$ was used and results were expressed in $\mu \mathrm{mol} \mathrm{Fe} \mathrm{F}^{2+} / \mathrm{mg}$ dry weight extract. The relative activity of the samples was compared with the standards ascorbic acid and butylated hydroxytoluene (BHT).

\section{DPPH radical assay}

The free radical scavenging activities of different extracts were carried out by using the 1,1-diphenyl-2-picrylhydrazyl (DPPH) assay (Braca et al. 2001). Crude extract $(0.1 \mathrm{~mL})$ was mixed with $3 \mathrm{~mL}$ of a $0.004 \%$ methanol solution of DPPH. The mixture were reacted in the dark condition for $30 \mathrm{~min}$ and the absorbance was determined at $517 \mathrm{~nm}$. The percentage inhibition activity was calculated using the following equation:

DPPH scavenging effect (The free radical scavenging $)=$ $\left[\left(\mathrm{Abs}_{\text {control }}-\mathrm{Abs}_{\text {sample }}\right) / \mathrm{Abs}_{\text {control }}\right] \times 100$, Where $\mathrm{Abs}_{\text {control }}$ is the absorbance of the control reaction, $\mathrm{Abs}_{\text {sample }}$ is the absorbance of the extract/standard.

\section{Statistical analysis}

Analysis of variance was performed by ANOVA. Means between treatment groups were compared for significance by using Duncan new multiple-range test. P values $<0.05$ were considered to be significant and $\mathrm{P}$ values $<0.01$ to be very significant. All experiments were performed in triplicate $(n=3)$ and results were reported as means \pm standard deviation (SD).

\section{Results}

\section{Isolation and identification of endophytic fungi}

A total of 216 tissue segments (including 54 roots, 54 stems, 54 leaves and 54 flowers) collected from 9 individuals of $S$. miltiorrhiza Bge.falba at the three sites were processed, and 14 endophytic fungi isolates were recovered. Of these, the majority $(\mathrm{n}=7, \mathrm{CF} 12.96 \%)$ was recorded from roots, followed by flowers $(n=5,9.26 \%)$, stems $(\mathrm{n}=1,1.85 \%)$ and leaves $(\mathrm{n}=1,1.85 \%)$, respectively (Table 1). 4 isolates mainly belonging to Alternaria sp. and Sterile mycelia were isolated from Site 1, 6 strains (Alternaria sp., Fusarium sp., and Sterile mycelia) were isolated from Site 2 and 4 strains (Alternaria sp., and Sterile mycelia) were isolated from Site 3. Among which, Alternaria sp. were the dominant species isolated 
Table 1 Genera and number of endophytic fungi recovered from root, stem, leaf and flower of $S$. miltiorrhiza Bge. f. alba

\begin{tabular}{lllll}
\hline Species & \multicolumn{4}{l}{$\begin{array}{l}\text { Number of isolates and Colonization frequency (\%) } \\
\end{array}$} \\
\cline { 2 - 5 } Sample tissues & Root & Stem & Leaf & Flower \\
& N (\%) & N (\%) & N (\%) & N (\%) \\
\hline Alternaria sp. & $0(0)$ & $0(0)$ & $1(1.85)$ & $5(9.26)$ \\
Fusarium sp. & $2(3.70)$ & $0(0)$ & $0(0)$ & $0(0)$ \\
Sterile mycelia & $5(9.26)$ & $1(1.85)$ & $0(0)$ & $0(0)$ \\
Total & $7(12.96)$ & $1(1.85)$ & $1(1.85)$ & $5(9.26)$ \\
\hline
\end{tabular}

from leaves and flowers, Sterile mycelia occurred in roots and stems, and Fusarium sp. were only present in roots. Six isolates of non-sporulating Sterile mycelia were grouped into two morphotypes (morphotypes SaR3, SaR4, SaR5, SaS1, and morphotypes SaR6, SaR7), which were both Basidiomycota as indicated by promoting sporulation. Moreover, the selected two non-sporulating fungi (SaR-3, SaR-6), Alternaria sp. (SaF-2), and Fusarium sp. (SaR-2) were further identified by the internal transcribed spacer (ITS) rRNA gene sequence analysis. The accession numbers were provided by the Genbank. The result of identification showed that two non-sporulating fungi SaR-3 (JQ409156) and SaR-6 (JQ409166) were closely related to Schizophyllum commune (EU530002) and Trametes hirsuta (EF546240) (Table 2), while SaF-2 (JQ409154) and SaR-2 (JQ409155) were closely related to Alternaria alternata (FJ228163) and Fusarium proliferatum (EF546240) (Table 2).

\section{Preliminary phytochemical screening}

Preliminary phytochemical screening of fungal ethanolic extracts showed the presence of saponins, flavonoids, cardiac glycosides, terpenoids, steroids, tannins, phenol, anthroquinone and alkaloids. The result of qualitative analysis of the phytochemicals were summarised in

Table 2 Identification of endophytic fungi based on sequence of ITS DNA

\begin{tabular}{|c|c|c|c|c|}
\hline Isolate & $\begin{array}{l}\text { Accession } \\
\text { number }\end{array}$ & $\begin{array}{l}\text { Size of ITS } \\
\text { amplicon (bp) }\end{array}$ & $\begin{array}{l}\text { Closest match } \\
\text { in GenBank }\end{array}$ & $\begin{array}{l}\text { Percentage } \\
\text { identity }\end{array}$ \\
\hline $\mathrm{SaF}-2$ & JQ409154 & 532 & $\begin{array}{l}\text { Alternaria alternata } \\
\text { (FJ228163) }\end{array}$ & 100 \\
\hline SaR-2 & JQ409155 & 518 & $\begin{array}{l}\text { Fusarium } \\
\text { proliferatum } \\
\text { (EF546240) }\end{array}$ & 99 \\
\hline SaR-3 & JQ409156 & 598 & $\begin{array}{l}\text { Schizophyllum } \\
\text { commune } \\
\text { (EU530002) }\end{array}$ & 99 \\
\hline SaR-6 & JQ409166 & 624 & $\begin{array}{l}\text { Trametes hirsuta } \\
\text { (EF546240) }\end{array}$ & 99 \\
\hline
\end{tabular}

Table 3. A. alternata SaF-2 and F. proliferatum SaR-2 showed presence of all of nine phytochemicals above, while S. commune SaR-3, T. hirsuta SaR-6 and plant extracts also had all the phytochemical except cardiac glycosides and anthraquinones. Cardiac glycosides was found to be present in the extracts of SaL-1, SaF-1, SaF-2, SaR-1, SaR-2, SaR-5, SaR-6 and SaS-1, while anthroquinone was found to be present in the extracts of SaF-2, SaF-3, SaR-2, SaR-5, SaR-6 and SaR-7. The presence of terpenoids and phenol was observed in all of the endophytes except Alternaria sp. SaF-5 and Schizophyllum sp. SaS-1, while flavonoids were found to be present in all the extracts except Alternaria sp. SaF-1 and Trametes sp. SaR-7. The results of phytochemical analysis indicated that the endophytic fungi from S. miltiorrhiza Bge.f.alba could produce the same or similar phytochemicals as those originally from the host plant, and new bioactive compounds that were not present in host plant.

\section{Determination of total phenol content and total flavonoid content}

The total phenolic content and total flavonoid content of four endophytic strains (A. alternata, F. proliferatum, $S$. commune and T. hirsuta) and host plant were expressed in terms of $\mathrm{mg}$ gallic acid equivalent (GAE)/g of extract and $\mathrm{mg}$ quercetin equivalents/g of extract respectively. The higher total phenolic content was found in the extract of F. proliferatum SaR-2 (21.75), followed by A. alternata SaF-2 extract (20.53) and Plant root extract (19.17)

Table 3 Phytochemical analysis for the ethanol extracts of different endophytic fungi and plant root

\begin{tabular}{|c|c|c|c|c|c|c|c|c|c|c|}
\hline Genera & Isolates & A & B & $C$ & $\mathrm{D}$ & $E$ & $F$ & G & $\mathrm{H}$ & 1 \\
\hline \multirow[t]{6}{*}{ Alternaria sp. } & SaL1 & - & + & + & + & + & + & + & - & - \\
\hline & SaF1 & + & - & + & + & & + & + & - & - \\
\hline & $\mathrm{SaF} 2$ & + & + & + & + & + & + & + & + & + \\
\hline & $\mathrm{SaF} 3$ & - & + & - & + & + & - & + & + & - \\
\hline & $\mathrm{SaF} 4$ & - & + & - & + & - & - & + & - & - \\
\hline & SaF5 & + & + & - & - & - & + & - & - & + \\
\hline \multirow[t]{2}{*}{ Fusarium sp. } & SaR1 & - & + & + & + & - & + & + & - & - \\
\hline & $\mathrm{SaR} 2$ & + & + & + & + & + & + & + & + & + \\
\hline \multirow[t]{4}{*}{ Schizophyllum sp. } & $\mathrm{SaR} 3$ & + & + & - & + & + & + & + & - & + \\
\hline & SaR4 & - & + & - & + & - & + & + & - & - \\
\hline & $\mathrm{SaR} 5$ & + & + & + & + & & + & + & + & - \\
\hline & SaS1 & - & + & + & - & + & - & - & - & + \\
\hline \multirow[t]{2}{*}{ Trametes sp. } & SaR6 & + & + & + & + & + & - & + & + & - \\
\hline & SaR7 & - & - & - & + & & + & + & + & - \\
\hline Plant root & & + & + & - & + & + & + & + & - & + \\
\hline
\end{tabular}

+: Presence, -: absence; Sa: Salvia miltiorrhiza Bge.f.alba; SaR, SaS, SaL and SaF: isolate assignment code from root, stem, leaf, and flower tissues respectively; A: Saponins, B: Flavonoids, C: Cardiac glycosides, D: Terpenoids, E: Steroids, F: Tannins, G: Phenol, H: Anthroquinone, I: Alkaloids. Data is three replicates of each sample. 
(Table 4). Similarly, the total flavonoid content was found to be higher in F. proliferatum SaR-6 (8.27), followed by $A$. alternata SaF-2 (7.36) and plant root (6.98) (Table 4). The more phenol and flavonoid content in the endophytic fungi than host plant may have contributed dramatically to their antioxidant activities.

\section{Antioxidant capacity analysis}

The antioxidant capacity of the ethanol extracts of four selected endophytes and plant extract were assessed for their ability to reduce TPRZ-Fe (III) complex to TPTZFe (II) (Table 4). The reducing ability of the ethanol extracts was in the range of 512.25 to $1682.21 \mu \mathrm{mol} \mathrm{Fe}$ (II)/mg. The FRAP values for the extracts of $F$. proliferatum SaR-2 and A. alternata SaF-2 were significantly higher than those of ascorbic acid and BHT, while the FRAP values for the extracts of $S$. commune SaR-3 and T. hirsuta SaR-6 were significantly lower than that of ascorbic acid but higher than that of BHT.

The antioxidant activities of the extracts of endophytic fungi and plant root, as compared with the standards ascorbic acid and BHT, were determined by the capability to scavenge DPPH free radicals. Figure 1 showed the dose response curve of DPPH radical scavenging activity of all samples. It was observed that the ethanol extracts of $F$. proliferatum SaR-2 and A. alternata SaF-2 had higher radical scavenging activity than that of plant root. At a concentration of $0.1 \mathrm{mg} / \mathrm{mL}$, the scavenging activity of the plant root extract reached $80.23 \%$, while the scavenging activities of $F$. proliferatum SaR-2 and A. alternata SaF-2 were $90.14 \%$ and $83.25 \%$ respectively. Even though the DPPH radical scavenging abilities of the extract of F. proliferatum SaR-2 (90.14\%) was lower than those of the standards ascorbic acid (95.43\%) and BHT $(94.14 \%)$ at $0.1 \mathrm{mg} / \mathrm{mL}$, it still reached at high concentration. Thus the study showed that $F$. proliferatum SaR2 could be a promising resource of natural antioxidants.

\section{Discussion}

During this study, an assessment was performed for the diversity of endophytic fungi from Salvia miltiorrhiza Bge. f.alba, which are only distributed in Shandong province of China and have been used for the treatment of various cardiovascular diseases (Zhou et al. 2012). A total of fourteen fungal endophytes were isolated and identified by the morphological and molecular method. Morphological investigations have resulted in the identification of two fungal species: Alternaria and Fusarium species. Six isolates belonging to non-sporulating fungi were identified on the basis of internal transcribed spacer (ITS) rRNA gene sequence analysis, which was consistent with the other reports from different hosts (Liu et al. 2007). The present study is the first report that several fungal endophytes are associated with $S$. miltiorrhiza Bge.f.alba.

It is interesting to record the predominance of Alternaria sp. from this host, as it was also dominant in many other reports from different host plants (Khan et al. 2010; Kumar et al. 2011; Liu et al. 2007). Besides Alternaria sp., other genera like Fusarium sp. were also reported frequently from a number of other plant species (Liu et al. 2007; Wang et al. 2008). Therefore, it appears that Alternaria and Fusarium species are able to associate endophytically with a wide range of host plants.

Phytochemical analysis was carried out to assess the diversity of chemical compounds produced by the endophytic fungi from S. miltiorrhiza Bge.f.alba. The results showed that there were significant amount of secondary metabolites including saponins, flavonoids, terpenoids, steroids, tannins, phenol, and alkaloids in the ethanolic extracts of the endophytic fungi, similar to or with more activity than those in the host plant root extracts. This result was consistent with the earlier reports (Govindappa et al. 2011; Sadananda et al. 2011). However, cardiac glycosides and anthraquinones were not present in the plant extracts but in the extracts of $A$. alternata SaF-2, $F$. proliferatum SaR-2, Schizophyllum sp. SaR-5 and T. hirsuta SaR-6. This result indicated that the fungal endophytes associated with S. miltiorrhiza Bge.f.alba had the capability of producing the same or similar phytochemicals as those present in the host plant, and new bioactive compounds that were not present in host plant. It is interesting to find that Fusarium sp. and Alternaria sp. could produce bioactive

Table 4 Total antioxidant activity and total phenolic content of ethanol extracts of four endophytic fungi and plant root

\begin{tabular}{llll}
\hline Samples & Phenol $(\mathbf{m g} / \mathbf{g})$ & Flavonoid $(\mathbf{m g} / \mathbf{g})$ & FRAP $(\boldsymbol{\mu m o l} \mathbf{l})$ \\
\hline Alternaria alternata SaF-2 & $20.53 \pm 0.08$ & $7.36 \pm 0.09$ & $1659.05 \pm 0.06$ \\
Fusarium proliferatum SaR-2 & $21.75 \pm 0.11$ & $8.27 \pm 0.12$ & $1682.21 \pm 0.05$ \\
Schizophyllum commune SaR-3 & $12.96 \pm 0.18$ & $5.62 \pm 0.15$ & $586.65 \pm 0.13$ \\
Trametes hirsuta SaR-6 & $11.21 \pm 0.25$ & $4.56 \pm 0.08$ & $512.25 \pm 0.15$ \\
Plant root extract & $19.17 \pm 0.09$ & $6.98 \pm 0.13$ & $1347.54 \pm 0.11$ \\
Ascorbic acid & & & $1655.25 \pm 0.07$ \\
BHT & & & $66.43 \pm 0.16$ \\
\hline
\end{tabular}

Data represent means of three replicates. 


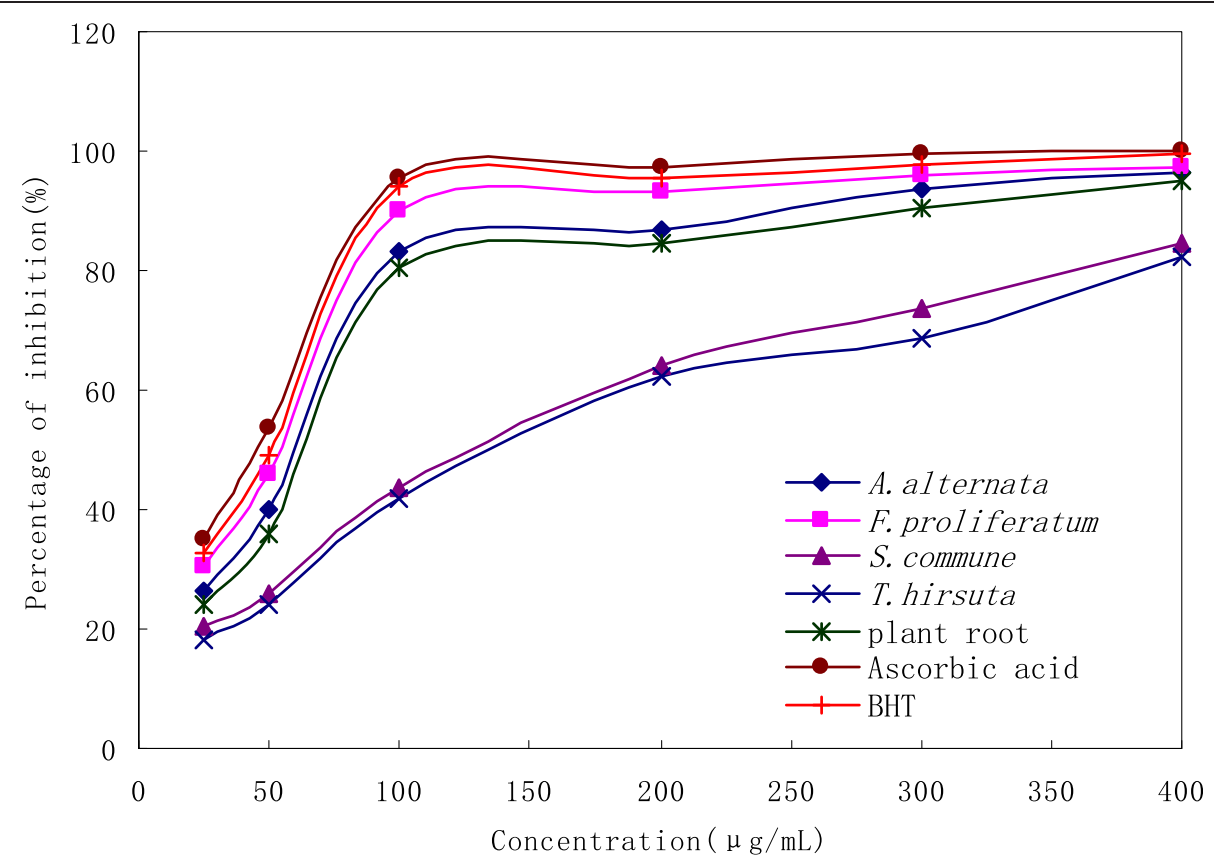

Figure 1 DPPH radical scavenging activity of the ethanol extracts of four selected endophytic fungi, plant root and the positive controls (ascorbic acid and BHT). All the experiments were repeated for three times.

compounds originally from their host plants. Similarly, Fusarium sp. and Alternaria sp. were found to produce active constituents such as paclitaxel, podophyllotoxin, camptothecine, which were also produced by their host plants (Zhao et al. 2011).

The presence of phytochemicals within fungal endophytes can be promising sources for medicinal or agrochemical use. The phytochemicals including phenols and flavonoids in fungal endophytes may be responsible for the antioxidant property (Hamilton et al. 2012). According to recent study, a highly positive correlation between total phenol content, total flavonoid content and antioxidant activity seems to be the trend in many endophytic fungi. In our study, strong antioxidant activities were present in the ethanol extracts of $F$. proliferatum SaR-2 and $A$. alternata SaF-2, comparable with the standards ascorbic acid and BHT. High phenolic and flavonoid content found in the ethanol extracts of Fusarium sp. and Alternaria sp. imply the contribution of these compounds to antioxidant activities, which was consistent with early study (Govindappa et al. 2011; Murthy et al. 2011; Sadananda et al. 2011). These findings indicate that endophytic fungi from S. miltiorrhiza Bge.f.alba may be effective as a promising potential for the development of novel antioxidant drugs. However, further studies are recommended to purify and characterize the structure of the biologically active constituents.

\section{Conclusions}

The present study demonstrated that fungal endophytes, Alternaria alternata SaF-2 and Fusarium proliferatum SaR-2 from Salvia miltiorrhiza Bge.f.alba yielded medically important phytochemical compounds. The antioxidant potential may be directly linked to the phenolic and flavonoid compounds present in the ethanol extracts of A. alternata SaF-2 and F. proliferatum SaR-2. To our knowledge, this is the first report that fungal endophytes associated with S. miltiorrhiza Bge.f.alba have been found to possess antioxidant potential.

\section{Abbreviations}

BHT: Butylated hydroxytoluene; CF: Colonization frequency; DPPH: 1,1-diphenyl2-picryl-hydrazyl; FRAP: Ferric ion reducing antioxidant power; GAEs: Gallic acid equivalents; ITS: Internal transcribed spacer; mg/g: Milligram/gram; PDA: Potato dextrose agar; ROS: Reactive oxygen species; Sa: Salvia miltiorrhiza Bge.falba; SaF: SaL, SaR and SaS, Flower, Leaf, Root and Stem tissues of Salvia miltiorrhiza Bge.f.alba; TPC: Total Phenolic Content; TPTZ: 2,4,6-tris (2-pyridyl)-S-triazine.

\section{Competing interests}

The authors declare that they have no competing interests.

\section{Authors' contributions}

$Y L$ took charge of experimental design, isolation and identification of endophytic fungi, phytochemical screening, interpretation of results, and write of the manuscript. XM performed the determination of antioxidant activity and interpretation of results. GP and RJ participated in the experiment design, ZY and JD analyzed data. ZM and GP involved in the correction of the paper. All the authors read and approved the final manuscript. 


\section{Acknowledgments}

This work was supported by National Natural Science Foundation of China (81403036, 81173489), Shandong Provincial Medical Science and Technology Development Project (2013WS0321), Shandong Provincial Natural Science Foundation (ZR2014HM048, ZR2010CM063), Tai'an Science and Technology Development Project (20132093) and Shandong Provincial Science and Technology Project of Population and Family Planning (2013-17).

\section{Author details}

${ }^{1}$ College of Life Sciences, Taishan Medical University, Tai'an 271016, China.

${ }^{2}$ College of Pharmacy, Taishan Medical University, Tai'an 271016, China.

${ }^{3}$ College of Chemical Engineering, Taishan Medical University, Tai'an 271016, China.

Received: 5 May 2014 Accepted: 15 March 2015

\section{Published online: 01 April 2015}

\section{References}

Aly AH, Debbab A, Kjer J, Proksch P (2010) Fungal endophytes from higher plants: a prolific source of phytochemicals and other bioactive natural products. Fungal Divers 41:1-16

Barros L, Calhelha RC, Vaz JA, Ferreira ICFR, Baptista P, Estevinho LM (2007) Antimicrobial activity and bioactive compounds of Portuguese wild edible mushrooms methanolic extracts. Eur Food Res Technol 225:151-156

Braca A, Tommasi ND, Bari LD, Pizza C, Politi M, Morelli I (2001) Natural antioxidants from plant material in phenolic compounds in food and their effects on health. J Nat Prod 64:892-895

Chen $\mathrm{H}$, Chen $\mathrm{F}$ (1999) Effects of methyl jasmonate and salicylic acid on cell growth and cryptotanshinone formation in Ti transformed Salvia miltiorrhiza cell suspension cultures. Biotechnol Lett 21:803-807

Devi NN, Prabakaran JJ, Wahab F (2012) Phytochemical analysis and enzyme analysis of endophytic fungi from Centella asiatica. Asian Pacific Journal of Tropical Biomedicine 2:1280-1284

Edeoga HO, Okwu DE, Mbaebie BO (2005) Phytochemical constituents of some Nigerian medicinal plants. Afr J Biotechnol 4:685-688

Finkel T, Holbrook NJ (2000) Oxidants, oxidative stress and biology of aging. Nature 408:239-247

Fisher PJ, Petrini O, Sutton BC (1993) A comparative study of fungal endophytes in leaves, xylem and bark of Eucalyptus in Australia and England. Sydowia 45:338-345

Jayanthi G, Kamalraj S, Karthikeyan K, Muthumary J (2011) Antimicrobial and antioxidant activity of the endophytic fungus Phomopsis sp. GJJM07 isolated from Mesua ferrea. Int J Curr Sci 1:85-90

Govindappa M, Bharath N, Shruthi HB, Santoyo G (2011) In vitro antioxidant activity and phytochemical screening of endophytic extracts of Crotalaria pallida. Free Rad Antiox 1:79-86

Hamilton CE, Gundel PE, Helander M, Saikkonen K (2012) Endophytic mediation of reactive oxygen species and antioxidant activity in plants: a review. Fungal Divers 54:1-10

Hao GP, Shi RJ, Wang JM, Qi B (2009) Construction of full-length cDNA library of white flower Salvia miltiorrhiza Bge f.alba root and partial EST sequence analysis. Afr J Biotechnol 8:2027-2035

Hao GP, Ji HW, Li YL, Shi RJ, Wang JM, Feng L, Huang LQ (2012) Exogenous ABA and polyamines enhanced salvianolic acids contents in hairy root cultures of Salvia miltiorrhiza Bge. f.alba. POJ 5:446-452

Khan R, Shahaz S, Choudhary MI, Khan SA, Ahmad A (2010) Communities of endophytic fungi in medicinal plant Withania somnifera. Pak J Bot 42:1281-1287

Kumar S, Kaushik N, Edrada-Ebel R, Ebel R, Proksch P (2011) Isolation, characterization, and bioactivity of endophytic fungi of Tylophora indica. World J Microbiol Biotechnol 27:571-577

Lachance PA, Nakat Z, Jeong WS (2001) Antioxidants: an integrative approach. Nutrition 17:835-838

Lam FF, Yeung JH, Chan KM, Or PM (2007) Relaxant effects of danshen aqueous extract and its constituent danshensu on rat coronary artery are mediated by inhibition of calcium channels. Vasc Pharmacol 46:271-277

Li LN (1997) Water soluble active components of Salvia miltiorrhiza and related plants. J Chin Pharm Sci 6:57-64

Lin X, Lu CH, Huang YJ, Zheng ZH, Su WJ, Shen YM (2007) Endophytic fungi from a pharmaceutical plant, Camptotheca acuminata: isolation, identification and bioactivity. World J Microbiol Biotechnol 23:1037-1040
Liu AH, Guo H, Ye M, Lin YH, Sun JH, Xu M, Guo DA (2007) Detection, characterization and identification of phenolic acids in Danshen using high-performance liquid chromatography with diode array detection and electrospray ionization mass spectrometry. J Chromatogr A 1161:170-182

Maobe MAG, Gatebe E, Gitu L, Rotich H (2013) Preliminary phytochemical screening of eight selected medicinal herbs used for the treatment of diabetes, malaria and pneumonia in Kisii Region, Southwest Kenya. Eur J Appl Sci 5:01-06

Murthy NK, Pushpalatha KC, Joshi CG (2011) Antioxidant activity and phytochemical analysis of endophytic fungi isolated from Lobelia nicotianifolia. J Chem Pharm Res 3:218-225

Plaza GA, Upchurch R, Brigmon RL, Whitman WB, Ulfig K (2004) Rapid DNA extraction for screening soil filamentous fungi using PCR amplification. Pol J Environ Stud 13:315-318

Sadananda TS, Nirupama R, Chaithra K, Govindappa M, Chandrappa CP, Vinay Raghavendra B (2011) Antimicrobial and antioxidant activities of endophytes from Tabebuia argentea and identification of anticancer agent (lapachol). J Med Plants Res 5:3643-3652

Saikkonen K, Faeth SH, Helander M, Sullivan TJ (1998) Fungal endophytes: a continuum of interactions with host plants. Annu Rev Ecol Syst 29:319-343

Schulz B, Boyle C, Draeger S, Römmert A, Krohn K (2002) Endophytic fungi:a source of novel biologically active secondary metabolites. Mycol Res 106:996-1004

Strobel G, Daisy B, Castillo U, Harper J (2004) Natural products from endophytic microorganisms. J Nat Prod 67:257-268

Szőllősi R, Szőllősi Varga I (2002) Total antioxidant power in some species of Labiatae (Adaptation of FRAP method). Acta Biologica Szegediensis 46:125-127

Taga MS, Miller EE, Pratt DE (1984) Chia seeds as a source of natural lipid antioxidants. J Am Oil Chem Soc 61:928-993

Tan RX, Zou WX (2001) Endophytes:a rich source of functional metabolites. Nat Prod Rep 18:448-459

Tomic A, Petrovic S, Pavlovic M, Trajkovski B, Milenkovic M, Vucicevic D, Niketic M (2009) Antimicrobial and antioxidant properties of methanol extracts of two Athamanta turbish subspecies. Pharm Biol 47:314-319

Verma VC, Kharmar RN, Strobel GA (2009) Chemical and functional diversity of natural products from plant associated endophytic fungi. Nat Prod Commun 4:1511-1532

Wang YT, LO HS, Wang PH (2008) Endophytic fungi from Taxus mairei in Taiwan: first report of Colletotrichum gloeosporioides as an endophyte of Taxus mairei. Bot Stud 49:39-43

Yan Q, Shi M, Ng J, Wu JY (2006) Elicitor-induced rosmarinic acid accumulation and secondary metabolism enzyme activities in Salvia miltiorrhiza hairy roots. Plant Sci 170:853-858

Zhang HW, Song YC, Tan RX (2006) Biology and chemistry of endophytes. Nat Prod Rep 23:753-771

Zhao J, Shan T, Mou Y, Zhou L (2011) Plant-derived bioactive compounds produced by endophytic fungi. Mini Rev Med Chem 11:159-168

Zhou L, Zuo Z, Chow MS (2005) Danshen: an overview of its chemistry, pharmacology, pharmacokinetics and clinical use. J Clin Pharmacol 45:1345-1359

Zhou X, Chan K, Yeung JHK (2012) Herb-drug interactions with Danshen (Salvia miltiorrhiza): a review on the role of cytochrome P450 enzymes. Drug Metabol Drug Interact 27:9-18

\section{Submit your manuscript to a SpringerOpen ${ }^{\circ}$ journal and benefit from:}

- Convenient online submission

Rigorous peer review

- Immediate publication on acceptance

- Open access: articles freely available online

- High visibility within the field

- Retaining the copyright to your article

Submit your next manuscript at springeropen.com 